\title{
Association of TNXB mutations with vesicoureteral reflux
}

A new study published in the Journal of the American Society of Nephrology reports that mutations in the TNXB gene, which encodes tenascin $\mathrm{XB}$, can cause vesicoureteral reflux (VUR).

"VUR is the most common type of congenital anomaly of the kidney and the urinary tract and kidney damage due to VUR is an important cause of endstage renal disease in children," says Rasheed Gbadegesin, corresponding author on the latest paper. "Although VUR has shown high heritability in both sibling and twin studies, the specific genetic causes have remained elusive owing to the lack of large pedigrees with adequate statistical power. This is the first time that a mutation in a gene encoding an extracellular matrix protein has been linked with the aetiology and pathogenesis of VUR."

Gbadegesin and colleagues identified a 97-member kindred with at least 16 individuals affected by VUR dating back at least five generations. Using sequential genome-wide linkage studies and wholeexome sequencing, they found a deleterious heterozygous mutation (T3257I) in the TNXB gene that segregated with the disease in the affected family. The variant was present in all affected individuals and in all obligate carriers and was absent from all unaffected members of the family.

"TNXB protein is expressed in both the refluxing and nonrefluxing ureter," says Gbadegesin. "A review of a publicly available database showed that TNXB is expressed in the ureter and the bladder of mice on embryonic day 15 , a period that coincides with the time when the ureterovesical junction is being formed."

The researchers went on to show that fibroblast cell lines carrying the T3257I mutant

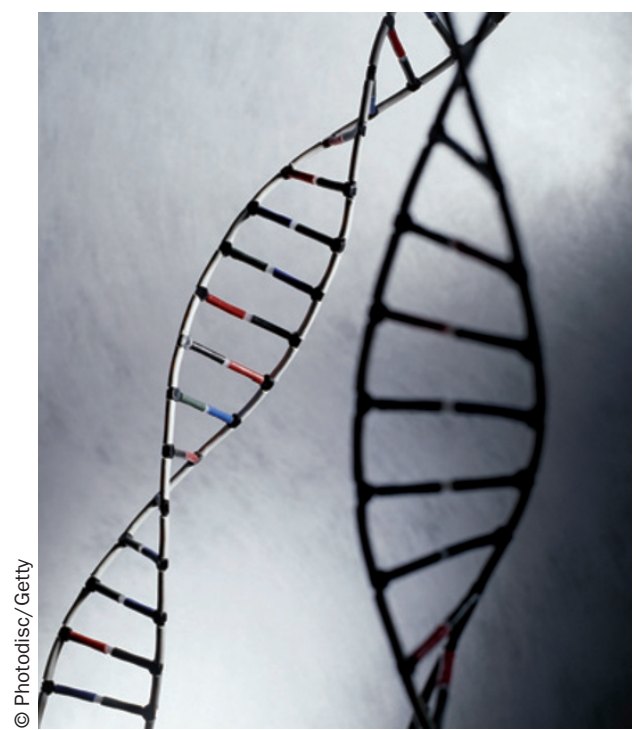

displayed reduced cell motility and expression of phosphorylated focal adhesion kinase. Immunohistochemical studies showed that TNXB is expressed in the human uroepithelial lining of the ureterovesical junction. According to Gbadegesin et al., these findings suggests that the T3257I mutation may alter integrin-mediated adhesion and dysregulate the extracellular matrix and tensile forces needed to close the ureterovesical junction as part of the anti-reflux mechanism.

"This finding opens up a whole different dimension and possible mechanism in understanding the pathophysiology of VUR," states Gbadegesin. "We plan to use knock-in mouse models of the variant identified in this study to explore the role of TNXB in the development of VUR and in kidney development generally. Identification of other VURassociated genes may lead to the development of noninvasive molecular diagnostic tools."

Rebecca Kelsey

Original article Gbadegesin, R. A. et al. TNXB mutations can cause vesicoureteral reflux. J.Am. Soc. Nephrol. doi:10.1681/ ASN.2012121148 